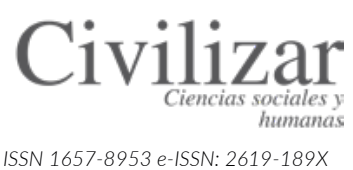

ISSN 1657-8953 e-ISSN: 2619-189X

\title{
Maltrato en relaciones de noviazgo de jóvenes universitarios en Bucaramanga y su área metropolitana*
}

\section{Abuse in courtship among young college students in Bucaramanga and its metropolitan area}

Jesús Redondo-Pachecos

Laura Viviana Durán-Cubides ${ }^{\ddagger}$ Marianela Luzardo-Briceño" Cándido José Inglés Saura

Artículo de investigación resultado del proyecto "Prevalencia y variables asociadas a la perpretación y la victimización de malos tratos en el noviazgo en adolescentes de Floridablanca, Santander" (Código 113-0118-3100) financiado por la Universidad Pontificia Bolivariana.

$\S$ Docente Investigador de la Universidad Pontificia Bolivariana. Doctor en Psicología. Correo electrónico:

jesus.redondo@upb.edu.co

(iD) 0000-0001-6063-1996

${ }^{\ddagger}$ Joven investigadora, egresada de la Universidad Pontificia Bolivariana. Correo:

vivis_duran15@hotmail.com

(iD) 0000-0003-2783-4497

"Docente Investigadora de la Universidad Pontificia Bolivariana. Doctora en Estadística.

Correo electrónico:

marianela.luzardo@upb.edu.co

(iD) 0000-0002-8364-5378

Docente Investigador de la Universidad Miguel Hernández de Elche, España.

Doctor en Psicología.

Correo electrónico: cjingles@umh.es

(iD) 0000-0001-9471-3551

Cómo citar:

Redondo-Pacheco, J., Durán-Cubides, L., Luzardo-Briceño, M., Inglés-Saura, C. (2020). Maltrato en relaciones de noviazgo de jóvenes universitarios en Bucaramanga y su área metropolitana. Civilizar: Ciencias Sociales y Humanas, $20(38)$, 53-64. https://doi.org/10.22518/jour. ccsh/2020.1a03

Recibido: $24 / 05 / 2019$

Aprobado: $24 / 02 / 2020$

Licencia Creative Commons

\section{Resumen}

El maltrato en las relaciones de pareja se evidencia a través de diferentes comportamientos agresivos que se presentan mediante múltiples particularidades (maltrato físico, psicológico, emocional, sexual y económico). El objetivo principal de esta investigación fue caracterizar las experiencias de maltrato presentes en las relaciones de noviazgo de jóvenes universitarios en Bucaramanga y su área metropolitana. La muestra estuvo conformada por 407 estudiantes universitarios vinculados a 26 carreras profesionales en 7 universidades públicas y privadas de la ciudad $(62.9 \%$ mujeres y $37.1 \%$ hombres), con edades comprendidas entre 18 y 30 años. El 91.6\% de los participantes informó haber vivenciado, al menos una vez, alguna de las tipologías de maltrato por parte de su pareja. Se evidenció que este es más frecuente hacia las mujeres; sin embargo, no se encontró una diferencia estadísticamente significativa en cuanto al género, exceptuando el maltrato físico. Se encontró mayor presencia de maltrato en los estudiantes entre los 21 y 22 años. Así mismo, el tipo de maltrato con mayor frecuencia fue el psicológico, seguido por el emocional, el físico, económico y, por último, el maltrato sexual.

\section{Palabras clave}

Maltrato, noviazgo, universitarios, género, edad.

\section{Abstract}

Abuse in couple relationships is evidenced through different aggressive behaviors that are presented through multiple peculiarities (physical, psychological, emotional, sexual and economic abuse). The main objective of this research was to characterize the experiences of mistreatment in the dating relationships of university students in Bucaramanga and its metropolitan area. The sample was made up of 407 university students linked to 26 professional careers in 7 public and private universities of the city (62.9\% women and $37.1 \%$ men), ages between 18 and 30. Of the total number of participants, 91.6\% reported having experienced, at least once, some type of abuse by their partner, evidencing that it is more frequent in women. However, no statistically significant difference was found in terms of gender, except for physical abuse. A greater presence of abuse was found in students between 21 and 22 years of age. Likewise, the most frequent type of abuse was psychological, followed by emotional, physical, economic and, finally, sexual abuse.

\section{Keywords}

Abuse, dating, university, gender, age. 


\section{Introducción}

La violencia o maltrato influyen significativamente en la salud pública. Este es un problema que afecta a toda la sociedad y que se manifiesta de múltiples formas (Foshee et al., 2010; Wolitzky-Taylor et al., 2008; Ybarra et al., 2016). Los estudios realizados en la última década frente a la presencia de violencia en las relaciones de noviazgo han revelado que esta tiene una frecuencia mucho mayor de lo que se creería. En consecuencia, múltiples autores han sugerido que la violencia en el noviazgo debería recibir la misma atención que aquella brindada a la violencia entre parejas maritales o en convivencia (Muñoz-Rivas et al., 2007; Rey-Anacona, 2009). El maltrato durante el noviazgo ha sido mucho menos estudiado que el maltrato dado dentro del matrimonio, tal vez por la creencia de que se precisa un nivel elevado de compromiso -como puede ser la convivencia, compartir gastos económicos o quizás tener hijos- para que se presenten actos violentos. No obstante, algunas investigaciones han indicado que en los noviazgos, la frecuencia del maltrato puede ser más elevada, aunque sus consecuencias generalmente no sean tan graves (Bonache et al., 2016; Exner-Cortens, 2014; Fernández-González et al., 2014; Hernando, 2007; Van de Bongardt et al., 2015).

Desde hace tiempo ha aumentado el interés por el estudio de las relaciones de pareja entre los jóvenes ya que se ha evidenciado cierta conexión entre estos tipos de violencia en la juventud y la posterior violencia en las parejas adultas (Matud, 2007; Sebastián et al., 2010). En este sentido, el Instituto Nacional de Medicina Legal y Ciencias Forenses (2015), en su boletín epidemiológico de información estadística de violencia contra la mujer, ha expuesto que son las relaciones entre jóvenes las que exhiben una mayor presencia de violencia. Específicamente, el rango de edad que posee un número significativamente más alto en cuanto a la vivencia de maltrato es de 25 a 29 años, con 1.414 casos reportados. En segundo lugar (con muy poca diferencia), se presenta un gran número de casos de violencia en las parejas en el rango entre 20 y 24 años, con 1.386 casos reportados para ese año. Además, otros estudios también han comprobado que las edades en las cuales se presenta un riesgo mayor de recibir maltrato por parte de la pareja son entre los 20 y los 24 años, siendo este un periodo que coincide precisamente con la etapa universitaria (Del Castillo et al., 2015; Guzmán-González et al., 2014; Tolan et al., 2006).
El maltrato entre parejas jóvenes ha cobrado tal importancia que, por ejemplo, la Organización Mundial de la Salud (2012) ha adelantado estudios acerca de la violencia en las relaciones amorosas de jóvenes universitarios y ha encontrado que esta es una situación que afecta a gran parte de la población. Otras investigaciones se han enfocado en conocer cuáles han sido las variables que se relacionan con este fenómeno y han encontrado que la violencia se relaciona con la baja autoestima del agresor, con conductas celotípicas y carencia de empatía (Adams y Cervantes, 2012; Olivera et al., 2012), además del uso excesivo de alcohol y la exposición a comportamientos violentos en los hogares en los primeros años de vida que permite que posteriormente se acepten y toleren ese tipo de actos. También se ha encontrado que la dependencia emocional hace parte de las variables relacionadas con la presencia de maltrato (Dejonghe et al., 2005; OMS, 2012).

Sin embargo, a pesar de la importancia que supone el maltrato en las relaciones de noviazgo, aún existe cierto desconocimiento al respecto. De hecho, en la legislación colombiana aún no existe un término que haga alusión a la violencia en el noviazgo. De manera que en sí, este no supone un delito. Aunque en caso de que se presente alguna denuncia, esta puede incluirse dentro de la violencia familiar.

Un aspecto importante por resaltar es que la frecuencia de la violencia es muy similar en hombres y en mujeres, especialmente en cuanto al maltrato psicológico. Incluso, el maltrato ejercido por las mujeres llega a superar al de los hombres. La mayoría de las investigaciones han demostrado que las tasas de prevalencia son muy similares entre hombres y mujeres, y que la violencia en las parejas jóvenes es bidireccional (Archer, 2000; Molidor y Tolman, 1998; Muñoz-Rivas et al., 2007; Watson et al., 2001; Strauss, 2004). Estos resultados indican que es necesario considerar, en ambos sexos, no solo la frecuencia de sujetos que exhiben conductas de maltrato, sino también la frecuencia de victimización.

El maltrato se presenta en diferentes manifestaciones; entre ellas, se destacan el maltrato físico, psicológico, sexual, emocional, y económico (Friedlander et al. 2013; Hamby, Finkelhor y Turner, 2012; Sabina et al. 2016; Temple et al., 2016). 
La terminología usada para referirse a la modalidad de maltrato determina la forma como se desarrollan las agresiones o comportamientos violentos, mas no hace referencia a las consecuencias que estos producen, puesto que tanto el maltrato físico, el sexual, el emocional, e incluso el económico, entre otros actos violentos, producen secuelas no solo físicas sino que, en la mayoría de las situaciones, también daño psicológico. Ahora bien, es primordial tener en cuenta que, a pesar de las creencias generalizadas que existen en torno a esta temática, el maltrato psicológico tiene consecuencias tan graves como el físico (Exner-Cortens et al., 2013; Morales y Rodríguez, 2012; Póo y Vizcarra, 2008; Rojas-Solís, 2011; Sebastián et al., 2010; Zúñiga et al., 2011; González, 2009).

La mayoría de las investigaciones se han centrado en la violencia física, probablemente, debido a su visibilidad y a los actos que acarrea; lo cual ha generado una visión objetiva de los actos y, por lo tanto, una alarma social. No obstante, este no es el único tipo de violencia que existe; también existe violencia psicológica, sexual, económica, entre otras. Este tipo de violencias, como se mencionó anteriormente, pueden ocasionar consecuencias para la salud iguales o aún más graves que la violencia física (Guzmán-González et al., 2014; Miller et al., 2013; Rey-Anacona, 2008; Rubio- Garay et al., 2015; Sarausa y Zubizarreta, 2000).

Ante este escenario, es muy importante analizar el maltrato en las relaciones de noviazgo sobre todo al considerar que sus consecuencias pueden ser considerables. Así, como ya se ha indicado, este tipo de comportamientos en las relaciones afectivas impactan notablemente debido a que pueden convertirse en modelos de conducta para una vida de pareja posterior (Krug et al., 2003). Por ello, y teniendo en cuenta que en nuestro contexto local no existen investigaciones relevantes frente a esta temática, consideramos que esta investigación ampliará la comprensión del fenómeno en cuestión.

\section{Metodología}

\section{Participantes}

En la investigación se tuvieron en cuenta como criterios de inclusión que los participantes fueran estudiantes universitarios en alguna de las universidades de Bucaramanga o de su área metropolitana (Colombia) y que se encontraran cursando entre el primer y el décimo semestre. La muestra se conformó con un total de 407 estudiantes universitarios de 26 carreras profesionales en 7 universidades públicas y privadas de la ciudad de Bucaramanga y su área metropolitana, aunque cabe indicar que no se consideró ni el semestre ni la carrera. El 62.9\% de los participantes mujeres, frente al $37.1 \%$ de los hombres, con edades entre los 18 y 30 años $(M=$ 20.6 años; $D E=2.171)$. Es importante destacar que los participantes debían estar en una relación de noviazgo en el momento del estudio o en el último año, hallando un promedio de duración de $\mathbf{2 2 . 2}$ meses en su relación actual o pasada.

El tipo de muestreo utilizado en la investigación fue no probabilístico a conveniencia, donde su elección se centra en las características de la investigación y el criterio del investigador (Hernández, Fernández y Baptista, 2014)). Además, la selección de los sujetos se debió por la conveniente accesibilidad a través de la técnica de muestreo incidental, donde se establece la intencionalidad de qué individuos harían parte de la muestra.

\section{Instrumentos}

En este estudio se usó la Lista de Chequeo de Experiencias de Maltrato en la Pareja -Forma A- (Rey-Anacona, 2009) y que fue realizada en Colombia. Se trata de un instrumento de carácter descriptivo y no psicométrico, que evalúa las formas de maltrato realizadas por la pareja, tanto de carácter físico, psicológico y emocional como económico, a través de 79 ítems, por medio de una escala tipo Likert. Para esta investigación, y considerando el objetivo y la población de estudio, sólo se utilizaron los primeros 68 ítems del instrumento, ya que los restantes se enfocan en personas que conformaran un hogar o tuvieran hijos.

Este instrumento fue validado "a nivel de contenido por jueces con titulación mínima de magister y experiencia investigativa en violencia intrafamiliar, quienes evaluaron la redacción de las instrucciones y los ítems y su pertinencia con respecto al tipo de violencia al que pertenecían" (Rey-Anacona, 2013, p. 145). El alfa de Cronbach en este estudio fue bastante alto (.95), lo que evidencia una significativa consistencia interna. Los resultados obtenidos se clasifican por frecuencia y tipo de maltrato. De este modo, se podrá evidenciar si los participantes han sido víctimas de las formas de maltrato planteadas. 


\section{Procedimiento}

El paso inicial consistió en establecer contacto con los participantes de las siete universidades seleccionadas para recoger la muestra, quienes fueron seleccionados mediante un muestreo no probabilístico por conveniencia. Una vez realizado el acercamiento a cada uno de ellos, se les presentó la investigadora y se les explicó el propósito de la investigación. Asimismo, se les expusieron los aspectos relacionados con el consentimiento informado, tales como el objetivo general del estudio, el tiempo de duración del instrumento, la estricta confidencialidad del estudio y, por supuesto, el derecho de aceptar o negar su participación en él. Una vez los convocados decidieron hacer parte de la investigación, se les dieron las indicaciones pertinentes para el desarrollo del instrumento Lista de chequeo de experiencias de maltrato en la pareja -Forma A y se atendieron las inquietudes generadas por este. Después de haber aplicado el instrumento pertinente a los 407 participantes, se procedió a construir una base de datos en Excel para, paso seguido, analizarla en el paquete estadístico SPSS versión 20.

\section{Resultados}

Del total de participantes, el 37.5\% fueron hombres $(\mathrm{n}=140)$ frente al $62.2 \%$ de mujeres $(n=232)$, y no se encontraron diferencias estadísticamente significativas entre géneros $\left(\mathrm{X}^{2}=1.072\right.$, $\mathrm{p}=.285)$. Asimismo, el 91.6\% $(\mathrm{n}=373)$ informó haber vivido alguna de las situaciones de violencia evaluadas por el instrumento de la investigación. Además, se pudo evidenciar que el tipo de maltrato más sufrido por los participantes fue el del tipo psicológico (91.4\%), seguido por el emocional (46.4\%), el físico (36.4\%), el económico (16.2\%) y, por último, el sexual (15.7\%).

Teniendo en cuenta los tipos de maltrato estudiados, se halló que las mujeres fueron quienes más sufrieron este tipo de conductas. La Tabla 1 muestra que donde más diferencia existe respecto al género fue el maltrato psicológico (Mujeres: 57.2\% y Hombres: $34.2 \%$ ), seguido del emocional (mujeres: $28.3 \%$ y hombres: $18.2 \%$ ), el físico (mujeres: $20.9 \%$ y hombres: $15.5 \%$ ), el sexual (mujeres: $9.3 \%$ y hombres: $6.4 \%$ ), y, finalmente, el económico (mujeres: 9.3\% y hombres: 6.9\%). Cabe indicar que no se encontraron diferencias estadísticamente significativas con respecto al género en la mayoría de los maltratos estudiados, salvo en el maltrato físico $(p=.084)$, en favor de las mujeres.

\section{Tabla 1}

Porcentaje de participantes por sexo que ejercieron conductas de cada tipo de maltrato

\begin{tabular}{|c|c|c|c|c|c|}
\hline Tipo de maltrato & Sexo & $\mathbf{n}$ & $\%$ & $\mathrm{X}^{2}$ & $\mathbf{p}$ \\
\hline \multirow{3}{*}{ Psicológico } & Hombre & 139 & 34.2 & \multirow{3}{*}{.130} & \multirow{3}{*}{.718} \\
\hline & Mujer & 233 & 57.2 & & \\
\hline & Total & 372 & 91.4 & & \\
\hline \multirow{3}{*}{ Emocional } & Hombre & 74 & 18.2 & \multirow{3}{*}{.637} & \multirow{3}{*}{.425} \\
\hline & Mujer & 115 & 28.3 & & \\
\hline & Total & 189 & 46.4 & & \\
\hline \multirow{3}{*}{ Físico } & Hombre & 63 & 15.5 & \multirow{3}{*}{2.979} & \multirow{3}{*}{.084} \\
\hline & Mujer & 85 & 20.9 & & \\
\hline & Total & 148 & 36.4 & & \\
\hline \multirow{3}{*}{ Económico } & Hombre & 28 & 6.9 & \multirow{3}{*}{.957} & \multirow{3}{*}{.328} \\
\hline & Mujer & 38 & 9.3 & & \\
\hline & Total & 66 & 16.2 & & \\
\hline \multirow{3}{*}{ Sexual } & Hombre & 26 & 6.4 & \multirow{3}{*}{.404} & \multirow{3}{*}{.525} \\
\hline & Mujer & 38 & 9.3 & & \\
\hline & Total & 64 & 15.7 & & \\
\hline
\end{tabular}

Fuente: Rey-Anacona, C. A. (2013). Prevalencia y tipos de maltrato en el noviazgo en adolescentes y adultos jóvenes. Terapia Psicológica, 31(2), 143-154. http://dx.doi.org/10.4067/S0718-48082013000200001 
En la Tabla 2 se observa el número y porcentaje de participantes que reportaron algún tipo de maltrato en cada una de las edades presentes. Se encontró que en cada una de estas, al menos un participante informó haber vivenciado como mínimo una de las situaciones descritas. Así, se halló que los porcentajes de maltrato en cada una de las edades son significativamente altos, superando el 90\% en muchos casos. En primer lugar, se encuentra el porcentaje relacionado con las edades de 26 , 27 y 30 años, que denota la vivencia de alguna de las conductas planteadas en un 100\% (aunque el número de participantes con estas edades es bajo). Asimismo, teniendo en cuenta los datos de los participantes, el rango de edades donde se encuentra mayor presencia de maltrato es entre los 21 y 22 años, con un porcentaje de $96 \%$ y $96.4 \%$ respec- tivamente. También es relevante destacar el 95.4\% para la edad de 19 años. Por último, las edades de 23 y 20 años también superan el 9o\% de presencia de maltrato en sus relaciones de noviazgo: un 91.7\% en la edad de 23 años y un $90.4 \%$ en la edad de 20 años.

A modo de conclusión, a partir de los ítems presentados se pudo evidenciar que el tipo de maltrato que se presenta con mayor frecuencia en las relaciones de noviazgo es el psicológico. Además, se encontró que las mujeres presencian mayor número de experiencias de maltrato que los hombres; aunque ambos géneros presentan en su mayoría diferentes tipos de maltrato. En el caso de las mujeres, estas sufren con más frecuencia maltrato psicológico, al contrario de los hombres que sufren más conductas relacionadas con el maltrato físico (ver Tabla 5).

Tabla 2

Número y porcentaje de participantes por edad que informaron al menos una conducta de maltrato

\begin{tabular}{|c|c|c|c|c|c|c|c|c|c|c|c|c|}
$\begin{array}{c}\text { Edad } \\
\begin{array}{c}\text { Total } \\
\text { participantes }\end{array}\end{array}$ & 87 & 66 & 52 & 76 & 56 & 24 & 17 & 24 & 3 & 1 & 1 \\
$\begin{array}{c}\text { Informaron } \\
\text { maltrato }\end{array}$ & 77 & 63 & 47 & 73 & 54 & 22 & 13 & 19 & 3 & 1 & 1 \\
\hline$\%$ por edad & 88.5 & 95.4 & 90.4 & 96 & 96.4 & 91.7 & 76.5 & 79.2 & 100 & 100 & 100 \\
\hline
\end{tabular}

Fuente: Rey-Anacona, C. A. (2013). Prevalencia y tipos de maltrato en el noviazgo en adolescentes y adultos jóvenes. Terapia Psicológica, 31(2), 143-154. http://dx.doi.org/10.4067/S0718-48082013000200001

Tabla 3

Diferencias por sexo en las conductas de maltrato ejercidas por los participantes

\begin{tabular}{|c|c|c|c|c|c|c|}
\hline \multirow[b]{2}{*}{ Maltrato informado } & \multicolumn{6}{|c|}{$M(D E)$} \\
\hline & $\begin{array}{l}\text { Tipo de } \\
\text { maltrato }\end{array}$ & General & Mujer & Hombre & $\mathbf{T}$ & $\mathbf{p}$ \\
\hline $\begin{array}{l}\text { 2. Le prohibió comprar objetos } \\
\text { para su uso personal. }\end{array}$ & Psicológico & $\begin{array}{c}.17 \\
(.539)\end{array}$ & $\begin{array}{l}.22 \\
(.613)\end{array}$ & $\begin{array}{l}.09 \\
(.372)\end{array}$ & 2.291 & .022 \\
\hline $\begin{array}{l}\text { 3. Puso a la familia de él (ella) o a } \\
\text { un miembro de dicha familia en su } \\
\text { contra }\end{array}$ & Psicológico & $\begin{array}{l}.29 \\
(.728)\end{array}$ & $\begin{array}{c}.33 \\
(.809)\end{array}$ & $\begin{array}{l}.21 \\
(.558)\end{array}$ & 1.701 & .090 \\
\hline $\begin{array}{l}\text { 7. Le humilló frente a otra(s) } \\
\text { persona(s). }\end{array}$ & Psicológico & $\begin{array}{l}.37 \\
(.725)\end{array}$ & $\begin{array}{c}.42 \\
(.763)\end{array}$ & $\begin{array}{l}.29 \\
(.649)\end{array}$ & 1.759 & .079 \\
\hline $\begin{array}{l}\text { 9. Controló sus actividades } \\
\text { cotidianas. }\end{array}$ & Psicológico & $\begin{array}{c}.84 \\
(1.047)\end{array}$ & $\begin{array}{c}.92 \\
(1.085)\end{array}$ & $\begin{array}{l}.70 \\
(.966)\end{array}$ & 2.117 & .035 \\
\hline $\begin{array}{l}\text { 14. Le persiguió cuando salió de la } \\
\text { casa o de otro sitio. }\end{array}$ & Psicológico & $\begin{array}{c}.32 \\
(.720)\end{array}$ & $\begin{array}{c}.39 \\
(.795)\end{array}$ & $\begin{array}{l}.19 \\
(550)\end{array}$ & 2.763 & .006 \\
\hline $\begin{array}{l}\text { 16. Mintió asegurando que usted } \\
\text { le iba a hacer daño. }\end{array}$ & Psicológico & $\begin{array}{l}.18 \\
(.523)\end{array}$ & $\begin{array}{l}.22 \\
(.580)\end{array}$ & $\begin{array}{l}.13 \\
(.405)\end{array}$ & 1.735 & .084 \\
\hline
\end{tabular}




\begin{tabular}{|c|c|c|c|c|c|c|}
\hline \multirow[b]{2}{*}{ Maltrato informado } & \multicolumn{6}{|c|}{$M(D E)$} \\
\hline & $\begin{array}{l}\text { Tipo de } \\
\text { maltrato }\end{array}$ & General & Mujer & Hombre & $\mathrm{T}$ & $\mathbf{p}$ \\
\hline $\begin{array}{l}\text { 17. Le culpó de todo lo malo que le } \\
\text { ocurre a él (ella). }\end{array}$ & Psicológico & $\begin{array}{l}.58 \\
(.917)\end{array}$ & $\begin{array}{l}.66 \\
(.988)\end{array}$ & $\begin{array}{c}.44 \\
(.762)\end{array}$ & 2.428 & .016 \\
\hline $\begin{array}{l}\text { 18. Le dijo que iba a agredir a un } \\
\text { miembro de su familia. }\end{array}$ & Emocional & $\begin{array}{l}.07 \\
(.354)\end{array}$ & $\begin{array}{l}.10 \\
(.418)\end{array}$ & $\begin{array}{c}.03 \\
(198)\end{array}$ & 1.965 & .050 \\
\hline $\begin{array}{l}\text { 19. Le arrojó algún objeto en un } \\
\text { momento de conflicto. }\end{array}$ & Físico & $\begin{array}{l}.21 \\
(.534)\end{array}$ & $\begin{array}{l}.16 \\
(.484)\end{array}$ & $\begin{array}{l}.30 \\
(.600)\end{array}$ & -2.729 & .007 \\
\hline $\begin{array}{l}\text { 20. Le apretó fuerte con intención } \\
\text { de lastimarla (lo). }\end{array}$ & Físico & $\begin{array}{l}.40 \\
(.768)\end{array}$ & $\begin{array}{l}.50 \\
(.859)\end{array}$ & $\begin{array}{c}.23 \\
(.544)\end{array}$ & 3.587 & .000 \\
\hline $\begin{array}{l}\text { 21. No le dejó salir de su casa (o la } \\
\text { de él o ella). }\end{array}$ & Psicológico & $\begin{array}{l}.30 \\
(.668)\end{array}$ & $\begin{array}{l}.36 \\
(.738)\end{array}$ & $\begin{array}{l}.19 \\
(.513)\end{array}$ & 2.457 & .014 \\
\hline $\begin{array}{l}\text { 23. Le golpeó con una parte del } \\
\text { cuerpo (con el puño, un pie, etc.). }\end{array}$ & Físico & $\begin{array}{l}.25 \\
(.636)\end{array}$ & $\begin{array}{l}.20 \\
(.614)\end{array}$ & $\begin{array}{l}.34 \\
(.664)\end{array}$ & -2.295 & .022 \\
\hline $\begin{array}{l}\text { 43. Le echó en cara lo que le había } \\
\text { brindado económicamente. }\end{array}$ & Psicológico & $\begin{array}{l}.43 \\
(.785)\end{array}$ & $\begin{array}{l}.50 \\
(.840)\end{array}$ & $\begin{array}{l}.32 \\
(669)\end{array}$ & 2.190 & .029 \\
\hline $\begin{array}{l}\text { 44. Involucró malintencionada- } \\
\text { mente a su familia o a la familia de } \\
\text { él (ella) en los conflictos de ambos. }\end{array}$ & Psicológico & $\begin{array}{l}.34 \\
(.751)\end{array}$ & $\begin{array}{l}.43 \\
(.832)\end{array}$ & $\begin{array}{l}.19 \\
(.562)\end{array}$ & 3.063 & .002 \\
\hline $\begin{array}{l}\text { 45. Llegó borracho (a) a su casa a } \\
\text { hacer escándalo. }\end{array}$ & Emocional & $\begin{array}{l}.24 \\
(.621)\end{array}$ & $\begin{array}{l}.30 \\
(.719)\end{array}$ & $\begin{array}{c}.14 \\
(.384)\end{array}$ & 2.556 & .011 \\
\hline $\begin{array}{l}\text { 47. Le echó de la casa de él (ella) o } \\
\text { la casa de ambos. }\end{array}$ & Psicológico & $\begin{array}{l}.21 \\
(.504)\end{array}$ & $\begin{array}{l}.16 \\
(.448)\end{array}$ & $\begin{array}{l}.28 \\
(.582)\end{array}$ & -2.346 & .019 \\
\hline $\begin{array}{l}\text { 51. Utilizó el sexo para castigarla } \\
\text { (o) o controlarla (o). }\end{array}$ & Psicológico & $\begin{array}{l}.14 \\
(.523)\end{array}$ & $\begin{array}{l}.05 \\
(.276)\end{array}$ & $\begin{array}{l}.30 \\
(.755)\end{array}$ & -4.806 & .000 \\
\hline $\begin{array}{l}\text { 52. Se refirió a asuntos pasados } \\
\text { para hacerla (lo) sentir mal. }\end{array}$ & Psicológico & $\begin{array}{c}.88 \\
(1.075)\end{array}$ & $\begin{array}{c}.95 \\
(1.102)\end{array}$ & $\begin{array}{c}.75 \\
(1.020)\end{array}$ & 1.802 & .072 \\
\hline $\begin{array}{l}\text { 57. Amenazó con hacerse daño a } \\
\text { sí mismo (a), si usted no hacía algo } \\
\text { que él (ella) dijo. }\end{array}$ & Emocional & $\begin{array}{l}.31 \\
(.722)\end{array}$ & $\begin{array}{l}.38 \\
(.798)\end{array}$ & $\begin{array}{l}.20 \\
(.554)\end{array}$ & 2.449 & .015 \\
\hline
\end{tabular}

Nota: Debido a la cantidad de ítems de la prueba sólo se expresan en la Tabla los ítems donde se hallaron diferencias estadísticamente significativas. Fuente: Rey-Anacona, C. A. (2013). Prevalencia y tipos de maltrato en el noviazgo en adolescentes y adultos jóvenes. Terapia Psicológica, 31(2), 143-154. http://dx.doi.org/10.4067/S0718-48082013000200001

\section{Discusión}

El objetivo general de la presente investigación fue caracterizar las experiencias de maltrato presentes en las relaciones de noviazgo de jóvenes universitarios en Bucaramanga y su área metropolitana. Los resultados del estudio evidencian un alto porcentaje de participantes que indicaron ser víctimas por parte de su pareja de, al menos, una de las conductas de maltrato estudiadas (91.6\%). Este dato genera gran preocupación debido a que se reitera lo obtenido en otras investigaciones, las cuales han expuesto porcentajes de violencia muy altos. De hecho, se han presentado cifras que superan el $80 \%$ en respuesta a haber experimentado como mínimo un evento de maltrato en sus relaciones de noviazgo (Pedraza-Banderas y Vega-Valero, 2015; Rey-Anacona, 2009; Samaniego y Freixas, 2010). Cabe resaltar que esta situación no ocurre en todos los casos; existen investigaciones que han arrojado datos contrarios, como la realizada por López-Cepero, Rodríguez-Franco, Rodríguez-Díaz, Bringas y Paíno (2015), quienes reportaron que "el 68.7\% de los y las participantes informaron no haberse sentido maltratados, atemorizados ni atrapados en la relación de pareja" (p. 64). Estos datos dejan un porcentaje menor de presencia de este fenómeno (31.3\%), aunque sigue siendo relativamente alto con respecto a la edad y el tipo de relación que poseen los participantes. 
Respecto a la edad, teniendo en cuenta que la muestra de participantes tenía edades comprendidas entre los 18 y 30 años, los mayores porcentajes de frecuencia con relación a estas experiencias de maltrato se evidenciaron en el rango de 19 a 23 años, especialmente entre los 21 y 22 años, con una incidencia superior al 95\%. Al respecto, diferentes investigaciones han presentado datos similares (Peña et al., 2013; Ramírez y Núñez, 2010; Redondo et al., 2017; Rubio-Garay et al., 2012) aunque no son exactos debido a que cada estudio posee una muestra específica con diferentes rangos de edad, según la conveniencia del estudio; lo que genera variaciones en este aspecto. Por ejemplo, Blázquez et al. (2011) llevaron a cabo su trabajo con una población de jóvenes universitarios con edades comprendidas entre 17 y 23 años y evidenciaron que a los 17 y los 18 años se presentaban mayores índices de violencia. Los autores concluyeron que existe relación entre el inicio temprano del maltrato y la forma como la violencia se puede desarrollar de manera severa y crónica en la edad adulta.

Además, en cuanto a los diferentes tipos de maltrato estudiados, los resultados indicaron que de las 68 conductas de maltrato evaluadas, fue en el psicológico donde se hallaron diferencias estadísticamente significativas, lo que evidencia que este es el tipo de maltrato más frecuente entre los jóvenes universitarios. Estos resultados concuerdan con los obtenidos en otras investigaciones, las cuales han encontrado que el maltrato psicológico presenta una mayor prevalencia frente a los demás tipos de maltrato (Pazos et al., 2014; Rojas-Solís y Carpintero, 2011; Samaniego y Freixas, 2010) y en investigaciones que también pretendían encontrar cuál es la forma de maltrato más común (Cortés-Ayala et al., 2015; Sebastián et al., 2010; Valdivia y González, 2014). Después del maltrato psicológico, en la presente investigación se encontró que el segundo tipo de maltrato más frecuente es el emocional, seguido por los abusos físicos, económicos y, en último lugar, los maltratos de carácter sexual -a diferencia de estudios similares que han constatado que los maltratos físicos y sexuales son más frecuentes y, posteriormente, los malos tratos psicológicos- (Rey-Anacona, 2013; Rivera et al., 2007; Sears et al., 2007).

Hasta el momento, muchas de las investigaciones realizadas en torno al maltrato entre parejas han tenido un enfoque de victimización femenina.
Dichos estudios han considerado los patrones machistas de la sociedad como una de las causas principales de este hecho (González y Fernández, 2010; López-Cepero, 2011; Sánchez et al., 2015); incluso a la hora de hacer referencia al maltrato en relaciones de pareja, lo plantean como violencia de género (Adam, 2013; Díaz et al., 2013; García-Díaz et al., 2013; Vázquez et al., 2010) -definida como todo acto de violencia basado en la pertenencia al sexo femenino que tenga o pueda tener como resultado un daño o sufrimiento físico, sexual o psicológico para la mujer como la ejercida por el hombre sobre su pareja mujer (Naciones Unidas, 1994)-. En este sentido, en muchos casos se está dando por hecho que tan solo los hombres son los victimarios y que en ningún caso las mujeres asumen el papel de agresoras; por ello, surge la necesidad de conocer las experiencias de maltrato en las relaciones de noviazgo desde una perspectiva bidireccional. En este sentido, se ha encontrado que tanto hombres como mujeres han sido víctimas y victimarios, lo cual también se demuestra en otras investigaciones (Celis-Sauce y Rojas-Solís, 2015; Cortés-Ayala et al., 2015; López-Cepero et al., 2015; Rojas-Solís, 2011).

También se hallaron diferencias respecto al género en las relaciones de noviazgo de los participantes. Así, se encontró que las mujeres fueron con mayor frecuencia víctimas de malos tratos en sus relaciones de pareja destacando el maltrato psicológico como el más sufrido. En este orden de ideas, estos resultados son contrarios a los de otras investigaciones que encontraron que son los hombres jóvenes los que más sufren este tipo de maltrato (López-Cepero et al., 2015; Rojas-Solís y Carpintero, 2011; Williams et al., 2008). Además, las experiencias de maltrato de los hombres están relacionadas con abusos psicológicos y físicos, aunque en menor frecuencia. Esto se relaciona con otros estudios que indican que las mujeres podrían presentar más disposición a usar la agresión física (Archer, 2000; Benavides, 2016; Pazos et al., 2014; Redondo, Luzardo, García e Inglés, 2017). A pesar de estos resultados, no se hallaron diferencias estadísticamente significativas en cuanto al género de los participantes, salvo en el maltrato físico; este hecho es afín con otros estudios que, de igual forma, no encontraron tales diferencias en torno al género (Muñoz-Rivas, Graña, y González, 2011; Rey-Anacona, 2009; Sears et al., 2007). 


\section{Conclusiones}

Las relaciones de adultos difieren sustancialmente de las establecidas por los jóvenes en su dinámica de poder, desarrollo de habilidades sociales e influencia de las parejas. Estos factores son fundamentales para comprender el maltrato en las relaciones de noviazgo y explican las tasas de perpetración que se evidencian en las estadísticas actuales.

Los hallazgos de este estudio sugieren que el maltrato en el noviazgo entre los universitarios en Bucaramanga y su área metropolitana puede ser un problema importante, lo que indica la necesidad de una mayor atención a este tema; además, reflejan la necesidad de implementar estrategias de prevención, detección e intervención, especialmente de formas sutiles de violencia para modificar las creencias de los jóvenes sobre el "amor ideal", considerando la influencia del género en el etiquetado de experiencias violentas.

Nuestro estudio presenta algunas limitaciones que deben tenerse en cuenta. Primero, el diseño transversal no permite contrastar relaciones causales. Otra limitación se relaciona con la forma como obtuvimos los datos, porque los cuestionarios pueden producir aquiescencia, incluso cuando son anónimos, lo que podría llevar a subestimar la prevalencia de conductas violentas.

En resumen, el maltrato en el noviazgo de los jóvenes se determinaría más por género que por la percepción de maltrato que estos tienen, y que podría estar condicionado por el desarrollo de un modelo social y cultural que difiere según al género (Muñoz-Rivas et al., 2011). Esto puede ser muy importante porque, en este grupo etario, es probable que la interpretación de los roles de los pares se vea más influenciada por valores imperantes de la sociedad que por la experiencia previa (García-Díaz et al., 2013). Por ello, son necesarios nuevos estudios sobre la violencia en el noviazgo de adolescentes y jóvenes que involucren el género porque la investigación científica se suele centrar más en las mujeres.

\section{Referencias}

Adams, J., y Cervantes, R. (2012). Violencia pasiva en mujeres universitarias. Un estudio exploratorio de las causas del deterioro de la autoestima. Psicología y Salud, 22(1) 133-139. http://psicologiaysalud. uv.mx/index.php/psicysalud/article/view/565/970

Adam, A. (2013). Una revisión sobre violencia de género. Todo un género de duda. Gaceta Internacional de Ciencia Forense, 9, 1-9. https://www.academia.
edu/download/44896589/4A1_Adam_GICF_o9. pdf

Archer, J. (2000). Sex differences in aggression between heterosexual partners: A meta-analytic review. Psychological Bulletin, 126(5), 651-68o. https://doi. org/10.1037/0033-2909.126.5.651

Benavides, J. (2016). Violencia en el Noviazgo: Diferencias de Género. Informes Psicológicos, 16(2), 27-36. http://dx.doi.org/10.18566/infpsicv16n2ao2

Blázquez, M., Moreno, J., y García-Baamonde, M. (2011). Desarrollo de la violencia psicológica durante el noviazgo en parejas de jóvenes universitarios/as. Apuntes de Psicología, 29(3), 397-412. http://www. apuntesdepsicologia.es/index.php/revista/article/ view/238

Bonache, H., Ramírez-Santana, G., y González-Méndez, R. (2016). Conflict resolution styles and teen dating violence. International Journal of Clinical and Health Psychology, 16(3), 276-286. https://doi. org/10.1016/j.ijchp.2016.03.003

Celis-Sauce, A., y Rojas-Solís, J. (2015). Violencia en el noviazgo desde la perspectiva de varones adolescentes. Informes Psicológicos, 15(1), 83-104. http:// dx.doi.org/10.18566/infpsicv15n1ao5

Córtes-Ayala, L., Flores, M., Bringas, C., Rodríguez-Franco, L., López-Cepero, J., y Rodríguez, F. (2015). Relación de maltrato en el noviazgo de jóvenes mexicanos. Análisis diferencial por sexo y nivel de estudios. Terapia Psicológica, 33(1), 5-12. https:// www.redalyc.org/articulo.oa?id=78539320001

Dejonghe, E., Bogart, G., Levendosky, A., Von Eye, A., y Davidson, W. (2005). Infant exposure to domestic violence predicts heightened sensitivity to adult verbal conflict. Infant Mental Health Journal, 26(3), 268-281. https://doi.org/10.1002/imhj.20048

Del Castillo, A., Hernández, M., Romero, A., e Iglesias, S. (2015). Violencia en el noviazgo y su relación con la dependencia emocional pasiva en estudiantes universitarios. Psicumex, 5(1), 8-18. https://doi. org/10.36793/psicumex.v5i1.248

Exner-Cortens, D., Eckenrode, J., y Rothman, E. (2013). Longitudinal associations between teen dating violence victimization and adverse health outcomes. Pediatrics, 131(1), 71-78. https://doi.org/10.1542/ peds.2012-1029

Exner-Cortens, D. (2014). Theory and teen dating violence victimization: Considering adolescent development. Developmental Review, 34(2), 168-188. https://doi.org/10.1016/j.dr.2014.03.001

Fernández-González, L., O’Leary, K. D., y Muñoz-Rivas, M. J. (2014). Age-related changes in dating aggression in Spanish high school students. Journal of Interpersonal Violence, 29(6), 1132-1152. https://doi. org/10.1177/0886260513506057 
Foshee, V., McNaughton Reyes, H., y Ennett, S. (2010). Examination of sex and race differences in longitudinal predictors of the initiation of adolescent dating violence perpetration. Journal of Aggression, Maltreatment \& Trauma, 19(5), 492-516. https://doi.org/10.1080/10926771.2010.495032

Friedlander, L. J., Connolly, J. A., Pepler, D. J., y Craig, W. M. (2013). Extensiveness and persistence of aggressive media exposure as longitudinal risk factors for teen dating violence. Psychology of Violence, 3(4), 310-322. https://doi.org/10.1037/aoo32983

García-Díaz, V., Fernández, A., Rodríguez, F., López, M., Del Pilar, M., y Lana, A. (2013). Violencia de género en estudiantes de enfermería durante sus relaciones de noviazgo. Atención Primaria, 45(6), 290296. https://doi.org/10.1016/j.aprim.2012.11.013

González, P. (2009). Violencia en las relaciones de noviazgo entre jóvenes $y$ adolescentes de la Comunidad de Madrid (Tesis doctoral). Universidad Complutense de Madrid, Madrid. http://eprints.ucm. es/8435/1/T30765.pdf

González, H., y Fernández, T. (2010). Género y maltrato: violencia de pareja en los jóvenes de Baja California. Estudios Fronterizos, 11(22), 97-128. http:// www.scielo.org.mx/pdf/estfro/v11n22/v11n22a4. pdf

Guzmán-González, M., García, N., Sandoval, S., Vásquez, B., y Villagrán, C. (2014). Violencia psicológica en el noviazgo en estudiantes universitarios chilenos: Diferencias en el apego y la empatía diádica. Revista Interamericana de Psicología, 48(3), 338-346. http://www.redalyc.org/pdf/284/28437897010. pdf

Hamby, S., Finkelhor, D., y Turner, H. (2012). Teen dating violence: co-occurrence with other victimizations in the National Survey of Children's Exposure to Violence (NatSCEV). Psychology of Violence, 2(2), 111-124. https://doi.org/10.1037/aoo27191

Hernández, R., Fernández, C., y Baptista, M. (2014). Metodología de la investigación. Mc Graw Hill Education.

Hernando, A. (2007). La prevención de la violencia de género en adolescentes. Una experiencia en el ámbito educativo. Apuntes de Psicología, 25(3), 325-340. http://www.apuntesdepsicologia.es/index.php/revista/article/view/128

Instituto Nacional de Medicina Legal y Ciencias Forenses (2015). Comportamiento de la Violencia de Pareja. Colombia, 2015. https://www.medicinalegal.gov. co/documents/20143/49523/Violencia+de+pareja. pdf
Krug, E. G., Dahlberg, L. L., Mercy, J. A., Zwi, A. B., y Lozano, R. (2003). Informe mundial sobre la violencia y la salud. Organización Mundial de la Salud. http:// doi.org/10.159o/Soo36-46652003000300014

López-Cepero, J. (2011). Victimización en el noviazgo de personas adolescentes y jóvenes hispanohablantes: Evaluación, prevalencia y papel de las actitudes (Tesis doctoral). Universidad de Sevilla. https://idus.us.es/xmlui/bitstream/handle/11441/26891/Y_TD_PS-PROV37.pdf?sequence=1

López-Cepero, J., Lana, A., Rodríguez-Franco, L., Paíno, S., y Rodríguez-Díaz, F. (2015). Percepción y etiquetado de la experiencia violenta en las relaciones de noviazgo juvenil. Gaceta Sanitaria, 29(1), 21-26. https://doi.org/10.1016/j.gaceta.2014.07.006

López-Cepero, J., Rodríguez-Franco, L., Rodríguez-Díaz, F., Bringas, C., y Paíno, S. (2015). Percepción de la victimización en el noviazgo de adolescentes y jóvenes españoles. Revista Iberoamericana de Psicología y Salud, 6(2), 64-71. https://doi.org/10.1016/j. rips.2015.04.001

Matud, M. (2007). Dating violence and domestic violence. Journal of Adolescent Health, 40(4), 295-297. https://doi.org/10.1016/j.jadohealth.2007.02.001

Miller, S., Williams, J., Cutbush, S., Gibbs, D., Clinton-Sherrod, M., y Jones, S. (2013). Dating violence, bullying, and sexual harassment: Longitudinal profiles and transitions over time. Journal of Youth and Adolescence, 42, 607-618. https://doi. org/10.1007/s10964-013-9914-8

Molidor, C., y Tolman, R. (1998). Gender and contextual factors in adolescent dating violence. Violence Against Women, 4(2), 180-194. https://doi. org/10.1177/1077801298004002004

Morales, N., y Rodríguez, V. (2012). Experiencias de violencia en el noviazgo de mujeres en Puerto Rico. Revista Puertorriqueña de Psicología, 23, 57-90. http://www.redalyc.org/pdf/2332/233224386oo3. pdf

Muñoz-Rivas, M., Graña, J., y González, P. (2011). Abuso psicológico en parejas jóvenes. Behavioral Psychology, 19(1), 117-131.

Muñoz-Rivas, M., Graña, J., O’Leary, K. D., y González, P. (2007). Aggression in adolescent dating relationships: Prevalence, justification, and health consequences. Journal of Adolescent Health, 40(4), 298-304. https://doi.org/10.1016/j. jadohealth.2006.11.137 
Naciones Unidas (1994). Declaración sobre la Eliminación de la Violencia contra la Mujer. http://www. ohchr.org/sp/ProfessionalInterest/Pages/ViolenceAgainstWomen.aspx

Olivera, J., Arias, J., y Amador, R. (2012). Tipos de violencia en el noviazgo: Estudiantes universitarias de la UAEM, Zumpango. Revista Electrónica de Psicología Iztacala, 15(1), 150-171.

Organización Mundial de la Salud. (2012). Centro de prensa. Organización Mundial de la Salud. https://apps.who.int/iris/bitstream/10665/98816/1/ WHO_RHR_12.36_spa.pdf?ua=1

Pazos, M., Oliva, A., y Hernando, A. (2014). Violencia en relaciones de pareja de jóvenes y adolescentes. Revista Latinoamericana de Psicología, 46(3), 148-159. http://www.redalyc.org/articulo. oa? id $=80533065002$

Pedraza-Banderas, G., y Vega-Valero, C. (2015). Las Estrategias de Afrontamiento ante la Violencia en el Noviazgo. Revista Digital Internacional de Psicología y Ciencia Social, 1(1), 133-140. https://doi. org/10.22402/j.rdipycs.unam.1.1.2015.31.133-140

Peña, F., Zamorano, B., Hernández, G., Hernández, M., Vargas, J., y Parra, V. (2013). Violencia en el noviazgo en una muestra de jóvenes mexicanos. Revista Costarricense de Psicología, 32(1), 27-40. http:// www.redalyc.org/articulo.oa? $\mathrm{id}=476748711003$

Póo, A., y Vizcarra, M. (2008). Violencia de pareja en jóvenes universitarios. Terapia psicológica, 26(1), 81-88. http://www.redalyc.org/articulo. oa? id $=78526107$

Ramírez, C., y Núñez, D. (2010). Violencia en la relación de noviazgo en jóvenes universitarios: un estudio exploratorio. Enseñanza e investigación en psicología, 15(2), 273-283.

Ramírez-Rivera, C. A., y Núñez-Luna, D. A. (2010). Violencia en la relación de noviazgo en jóvenes universitarios: un estudio exploratorio. Enseñanza e Investigación en Psicología, 15(2), 273-283. http:// www.redalyc.org/pdf/292/29215980o03.pdf

Redondo, J., Inglés, C. J., y García, K. L. (2017). Papel que juega la edad en la violencia en el noviazgo de estudiantes de la Universidad Pontificia Bolivariana de Bucaramanga. Diversitas: Perspectivas en Psicología, 13(1), 41-54. https://www.redalyc.org/ jatsRepo/679/67952833003/index.html

Redondo, J., Luzardo, M., García-Lizarazo, K.L., e Inglés, C.J. (2017). Malos tratos durante el noviazgo en jóvenes universitarios: diferencias de sexo. Revista $I+D$ - Universitaria de Investigaciones y DesarrolloUDI, 9(1), 59-69. https://doi.org/10.33304/revinv. vo9n1-2017006
Rey-Anacona, C. A. (2008). Prevalencia, factores de riesgo y problemáticas asociadas con la violencia en el noviazgo: una revisión de la literatura. Avances en Psicología Latinoamericana, 26(2), 227-241. http:// www.redalyc.org/articulo.oa?id=79926209

Rey-Anacona, C. A. (2009). Maltrato de tipo físico, psicológico, emocional, sexual y económico en el noviazgo: Un estudio exploratorio. Acta colombiana de psicología, 12(2), 27-36. http://www.redalyc. org/articulo.oa?id=79815640003

Rey-Anacona, C. A. (2013). Prevalencia y tipos de maltrato en el noviazgo en adolescentes y adultos jóvenes. Terapia psicológica, 31(2), 143-154. http://dx.doi. org/10.4067/So718-48082013000200001

Rivera, L., Allen, B., Rodríguez, G., Chávez, R., y Lazcano, E. (2007). Prevalence and correlates of adolescent dating violence: Baseline study of a cohort of 7960 male and female Mexican public school students. Preventive Medicine, 44(6), 477-484. https://doi. org/10.1016/j.ypmed.2007.02.020

Rojas-Solís, J. (2011). Violencia de pareja en universitarios españoles: resultados preliminares de un estudio exploratorio. International Journal of Developmental and Educational Psychology, 5(1), 571-581. http://www.redalyc.org/articulo. oa? id=349832343062

Rojas-Solís, J., y Carpintero, E. (2011). Sexismo y agresiones físicas, sexuales y verbales-emocionales, en relaciones de noviazgo de estudiantes universitarios. Electronic Journal of Research in Educational Psychology, 9(2), 541-564. http://www.redalyc. org/articulo.oa? $\mathrm{id}=293122840004$

Rubio-Garay, F., Carrasco, M., Amor, P., y López-González, M. (2015). Factores asociados a la violencia en el noviazgo entre adolescentes: una revisión crítica. Anuario de Psicología Jurídica, 25(1), 47-56. https:// doi.org/10.1016/j.apj.2015.01.001

Rubio-Garay, F., López-González, M., Saúl, L., y SánchezElvira-Paniagua, A. (2012). Direccionalidad y expresión de la violencia en las relaciones de noviazgo de los jóvenes. Acción Psicológica, 9(1), 61-70. https://doi.org/10.5944/ap.9.1.437

Sabina, C., Cuevas, C., y Cotignola-Pickens, H. (2016). Longitudinal dating violence victimization among Latino teens: Rates, risk factors, and cultural influences. Journal of Adolescense, 47, 5-15. https:// doi.org/10.1016/j.adolescence.2015.11.003

Samaniego, E., y Freixas, A. (2010). Estudio sobre la identificación y vivencia de violencia en parejas adolescentes. Apuntes de Psicología, 28(3), 349366. http://www.apuntesdepsicologia.es/index. php/revista/article/view/224 
Sánchez, M., Martín, A., y Palacios, B. (2015). Indicadores de violencia de género en las relaciones amorosas. Estudio de caso en adolescentes chilenos. Pedagogía Social, 26, 85-109. https://recyt.fecyt.es/index. php/PSRI/article/view/38444

Sarasua, B., y Zubizarreta, I. (2000). Violencia en la pareja. Ediciones Aljibe.

Sears, H., Byers, E., y Price, E. (2007). The co-occurrence of adolescent boys' and girls' use of psychologically, physically, and sexually abusive behaviours in their dating relationships. Journal of Adolescence, 30(3), 487-504. https://doi.org/10.1016/j.adolescence.2006.05.002

Sebastián, J. Ortiz, B., Gil, M., Gutiérrez, M., Hernáiz, A., y Hernández, J. (2010). La Violencia en las Relaciones de Pareja de los Jóvenes. ¿Hacia Dónde Caminamos? Clínica contemporánea, 1(2), 71-83. https:// doi.org/10.5093/cc2010v1n2a1

Straus, M. (2004). Prevalence of violence against dating partners by male and female university students worldwide. Violence Against Women, 10(7), 790811. https://doi.org/10.1177/1077801204265552

Temple, J., Choi, H., Elmquist, J., Hecht, A., Miller-Day, M., Stuart, G., Brem, M., y Wolford-Clevenger, C. (2016). Psychological Abuse, Mental Health, and Acceptance of Dating Violence Among Adolescents. Journal of Adolescent Health, 59(2), 197-202. https://doi.org/10.1016/j.jadohealth.2016.03.034

Tolan, P., Gorman-Smith., y Henry. D. (2006). Family Violence. Annual Review of Psychology, 57(1), 557-583. https://doi.org/10.1146/annurev. psych.57.102904.190110

Valdivia, M., y González, L. (2014). Violencia en el noviazgo y pololeo: una actualización proyectada hacia la adolescencia. Revista de Psicología, 32(2), 330-355. http://www.redalyc.org/articulo. oa?id $=33783261800$

Van de Bongardt, D., Yu, R., Dekovi'c, M., y Meeus, W. H. J. (2015). Romantic relationships and sexuality in adolescence and young adulthood: The role of parents, peers, and partners. European Journal of Developmental Psychology, 12(5), 497- 515. https:// doi.org/10.1080/17405629.2015.1068689
Vázquez, F., Torres, A., Otero, P., Blanco, V., y López, M. (2010). Prevalencia y factores de riesgo de la violencia contra la mujer en estudiantes universitarias españolas. Psicothema, 22(2), 196-201. http:// www.redalyc.org/articulo.oa?id $=727124960$

Watson, J., Cascardi, M., Avery-Leaf, S., y O’Leary, K., (2001). High school students' responses to dating aggression. Violence and Victims, 16(3), 339-348.

Williams, J., Ghandour, R., y Kub, J. (2008). Review female perpetration of violence inheterosexual intimate relationships: adolescence through adulthood. Trauma Violence \& Abuse, 9(4), 227-249. https://doi.org/10.1177/1524838008324418

Wolitzky-Taylor, K., Ruggiero, K., Danielson, C., Resnick, H., Hanson, R., Smith, D., Saunders, B., y Kilpatrick, D. (2008). Prevalence and correlates of dating violence in a national sample of adolescents. Journal of the American Academy of Childand Adolescent Psychiatry, 47(7), 755-762. https://www.ncbi. nlm.nih.gov/pmc/articles/PMC3485067/

Ybarra, M., Espelage, D., Langhinrichsen-Rohling, J., Korchmaros, J., y Boyd, D. (2016). Lifetime Prevalence Rates and Overlap of Physical, Psychological, and Sexual Dating Abuse Perpetration and Victimization in a National Sample of Youth. Archives of Sexual Behavior, 45, 1083-1099. https://doi. org/10.1007/s10508-016-0748-9

Zúñiga, M., Martínez, P., Hernández, I., De Valle, M., y López, M. (2011). Violencia durante el noviazgo. Desarrollo científico de enfermería, 19(7), 242-245. http://www.index-f.com/dce/19/r19-242.php 
